www.jmscr.igmpublication.org

Impact Factor 5.84

Index Copernicus Value: 83.27

ISSN (e)-2347-176x ISSN (p) 2455-0450

crossref DOI: _https://dx.doi.org/10.18535/jmscr/v5i3.190

Journal Of Medical Science And Clinical Research

\title{
Efficacy and Safety of a Combination of Paracetamol, Levocetirizine and Phenylephrine in the symptomatic treatment of Common Cold and Allergic Rhinitis in Children: Phase IV Clinical Study
}

\author{
Authors \\ Dr Mayuresh Kiran ${ }^{1}$, Shruthi George ${ }^{2}$, Lalit Pawaskar ${ }^{3}$ \\ ${ }^{1}$ General Manager, Medical Services, Centaur Pharmaceuticals Pvt. Ltd. \\ ${ }^{2}$ Officer, Medical Services, Centaur Pharmaceuticals Pvt. Ltd. \\ ${ }^{3}$ Research Associate, Pharmacovigilance, Centaur Pharmaceuticals Pvt. Ltd. \\ Corresponding Author \\ Dr. Mayuresh Kiran \\ General Manager, Medical Services, Centaur Pharmaceuticals Pvt. Ltd., Mumbai, India. \\ Mobile No. +919820068567 \\ Email: mayuresh_kiran@rediffmail.com
}

\begin{abstract}
Introduction -It is the most frequently encountered self-limiting viral respiratory diseases. Common cold has a greater adverse impact on, absenteeism, productivity and daily life so symptomatic therapy to treat the symptoms is often employed. A FDC of Paracetamol, an antipyretic, Phenylephrine, a nasal decongestant and Levocetirizine, an anti-histaminic is popular in the treatment of Common Cold and Allergic Rhinitis in adults. This Phase IV study appraised the efficacy and safety of a combination of Paracetamol, Phenylephrine and Levocetirizine in treatment of common cold.

Methodology-Of 185 enrolled, 156 patients completed the study. Safety assessment was made by inspecting the adverse events during trial. Efficacy assessment was made by decrementing in TSS and four point Likerttype scales.

Results- Reduction in TSS from 5.05 (baseline) to 2.58 (day 3) and 0.61(day 5). One point reduction in Likert-type symptom scale from Moderate to Mild took just 3 days. Nearly all the patients had $>50 \%$ reduction in symptom score at all visit and majority of patients had complete relief from the symptom. 20 episodes of adverse events occurred and were of mild intensity.

Conclusion - A fixed-dose combination of Paracetamol, Phenylephrine and Levocetirizine is safe and effective in the treatment of Common Cold and Allergic Rhinitis in children.

Keywords - Paracetamol, Levocetirizine, Phenylephrine, Common Cold, Allergic Rhinitis.
\end{abstract}

\section{Introduction}

Worldwide, common cold is the most frequently encountered self-limiting viral respiratory diseases. According to Sir William Osler, the only way to treat Common Cold is with contempt; it still remains the leading cause of visits to the Doctor ${ }^{1}$.As per the United States Attitudes of Consumers toward Health, Cough, and Cold (ACHOO) survey, common cold has a greater adverse impact on productivity, absenteeism and 
daily life ${ }^{2}$. The definitions of common cold are rather vague. It is an acute viral infection of the upper respiratory tract involving the nose, sinuses, pharynx and larynx caused by one of several respiratory viruses, most commonly the rhinovirus.These viruses which concentrate in nasal secretions are easily transmitted through coughing, sneezing or blowing the nose. Common cold is symptomatologically diagnosed with fever, cough, rhinorrhoea, nasal congestion, sore throat, headache, and myalgia being the most common symptoms $^{3}$.

Allergic rhinitis is a heterogeneous disorder often undiagnosed despite high ubiquity. About 20$30 \%$ of the Indian population suffers from allergic rhinitis ${ }^{4}$. Recently, the document Allergic Rhinitis and its Impact on Asthma (ARIA), developed in collaboration with the WHO depicted how a disease so frequently affecting human race is way more disastrous and its complications and sequelae should be the subject of extensive research ${ }^{5}$. Allergic rhinitis if not treated, leads to acute asthma, thereby resulting in hospitalization of the patient. A survey by all India co-ordinated project on Aeroallergens and human health, New Delhi, 2000, cited that of the $20-30 \%$ of the population suffering from Allergic Rhinitis, $15 \%$ are likely to develop Asthma ${ }^{4}$.

Generally caused by viruses, the common cold is treated symptomatically. The signs and symptoms of common cold overlap with those of other conditions likeallergic rhinitis presenting similarly. Monotherapy may be scanty to relieve all the symptoms of common cold and allergic rhinitis, thence multiple drug combinations subsuming an Antihistamine, Analgesic/Antipyeretic/Anti inflammatory and Nasal Decongestants are used to treat these varieties of Symptoms ${ }^{6}$.

Paracetamol is a non-steroidal anti-inflammatory drug, most commonly used centrally acting analgesic and its use as an antipyretic to treat fever, headache and body ache are ubiquitous ${ }^{7}$. Paracetamol does not alter acid base balance, depress respiration or cause gastric irritation.Levocetirizine, the R-enantiomer of
Cetirizine is a non-sedating, new generation antihistamine with high affinity for and occupancy of the H1-receptor ${ }^{8}$.It blocks the effects of histamine, key mediator of allergic inflammation, which causes runny nose, itching, sneezing and watery eyes. It has abridged anticholinergic and antiserotonin effects and therefore is singled out in patients with allergy since it does not over dry respiratory secretions ${ }^{9}$.Phenylephrine is a sympathomimetic primarily used as a systemic nasal decongestant ${ }^{6}$. The mechanism by which decongestants bring off their action is by activation of postjunctional alpha 1-adrenergic receptors found on precapillary and post capillary blood vessels of the nasal mucosa. Activation of these receptors by distinct binding of the sympathomimetic agent to the binding site of the receptor or by enhanced release of norepinephrine leads to vasoconstriction. Such vasoconstriction leads to shrinkage of the tissue by decreasing blood flow through the nasal mucosa ${ }^{7}$.

Cochrane meta-analysis assessed combination of antipyretic, decongestant and antihistaminic for the symptomatic treatment of the common cold in children $^{10}$.

Eccles et al. ${ }^{11}$, stated that multi-ingredient combination products for multi-symptom relief are formulated to simply, safely and simultaneously treat multiple symptoms when used as directed in adults and children.

A combination of Paracetamol, Levocetirizine and Phenylephrine is readily available and studied for the treatment of common cold and allergic rhinitis in adults. However due to the inadequacy of clinical data available for this combination in children, a phase IV study was conducted to document the efficacy and safety of the combination ofParacetamol, Levocetirizine and Phenylephrine in the treatment of common cold and allergic rhinitis in children.

\section{Methodology}

This phase IV clinical trial enrolled 12 Pediatricspecialty centers in various cities in India for this study. This study was conducted 
fromNovember 2016 to February 2017. A total of 185 patients were recruited for the study, out of which 156 patients completed. 29 patients were lost to followup.

\section{Inclusion and Exclusion Criteria}

The study subsumed patients of both gender between the age 6 years to 11 years .Patients included in the study were diagnosed of common cold having 4 out of the 9 symptoms of headache, fever, bodyache , nasal congestion, rhinorrhoea, sneezing, sore throat, dysphonia and malaise) extant for not more than 48 hours were embodied in the study. Only the patients who would strictly cohere to the protocol were recruited for the study. Patients with any intricacy due to common cold like otitis media, tonsillitis, sinusitis etc. were excluded from the study since that avail use of antibiotics also would prolong the treatment duration. Patients with hypersensitivity to the individual study drugs, patients having hepatic and renal impairment were also excluded as Paracetamol is present in the study drug. Patients having blood pressure of more than $130 / 90 \mathrm{~mm}$ were refrained from the study as Phenylephrine which is present in the study drug can result in vasoconstriction causing increase in BP.

\section{Study Intervention}

Study drug - A combination of Paracetamol $250 \mathrm{mg}+$ Levocetirizine Hydrochloride $1.25 \mathrm{mg}+$ Phenylephrine Hydrochloride $5 \mathrm{mg}$ per $5 \mathrm{ml}$ was provided by the sponsor free of cost to the patient enrolled in the study. Study dosage and administration - Patients and Guardians were instructed to take the dose of $5 \mathrm{ml}$ thrice a day for a study period of 5 days, after food (Breakfast, Lunch\& Dinner) with a glass of water.

\section{Study Procedure}

The study stretch was decided to be 5 days. Patients of common cold and allergic rhinitis satiating the inclusion and exclusion criteria were recruited for the study. Medical history was taken and physical examination (including the vital signs, systemic and general examination) was conducted by the investigators. Patients were given free samples in the dose of $5 \mathrm{ml}$ twice a day by the investigator.Patients were asked to maintain a symptoms diary and note any adverse events occurring during the study duration. Three visits were outlined for the patients recruited in this study - V0 (baseline visit) on day 1, V1 (reevaluation visit) on day 3 and V2 (conclusion visit) on day 5. Total Symptom Score (TSS) and adverse events occurring were esteemed during each visit along with medical history and physical examination. Investigators were asked to desist the study drug in case of severe adverse event and with discretion, clinical experience contingent upon mild to moderate adverse events.

\section{Concomitant Therapy}

No Pharmacological intervention and medication including, topical decongestants (sprays/ drops and aromatic oils), antibiotics, multi-vitamins and multiminerals were allowed during the study duration, other than study drug.

Non-Pharmacological interventions like drinking of warm/hot water at regular intervals and steam inhalation were allowed and encouraged during the study.

\section{Efficacy Assessment}

The primary assessment was reduction in Total Symptom Score (TSS) which was a score of all the symptoms on an eleven-point scale (0 to 10) where 0 is no symptoms and 10 is maximum tolerated symptoms. The TSS was further extrapolated to the Likert-type symptom severity scale with 4 grades - no symptoms (0 on TSS), mild ( $1-4$ on TSS), Moderate (5 - 8 on TSS) and Severe (9 - 10 on TSS). The secondary assessment was number of patients having no symptoms ( 0 on TSS) on day 5 and number of patients having more than $50 \%$ reduction in TSS.

\section{Safety Assessment}

Patients were questioned for any adverse event and the same if present was noted in the case record form during each post-dose visit. 
Patients with any adverse events if present were recorded in the case report form after thorough investigation. These adverse events were categorized into non-serious adverse events and serious adverse events. Naranjo's scale of probability was used to classify the adverse event as non-drug related or drug related. Adverse events were followed up by the investigators till the symptoms subside.

\section{Regulatory and Ethical Matters}

The study drug in combination has been approved for manufacturing and marketing in 2005 (with minor change in composition). The forenamed combination is available under various brands but is classified as schedule $\mathrm{H}$ drug in India, i.e. to be sold in presence of prescription of registered medical practitioners only. Patients engaged voluntarily read and signed the informed consent form. The protocol, informed consent form, case record form, investigators $\mathrm{CV}$, investigators undertaking, investigators medical registration certificates (including post-graduation certificates) and ethics committee registration certificates were submitted to the office of Drug Controller General of India (DCGI), Central Drugs Standard Control Organization (CDSCO) and are registered under ref. no. 29395/16.

The protocol of the study was approved by the institutional ethics review board and with the 1964 Helsinki declaration and its later amendments or comparable ethical standards.

\section{Results}

A total of 185 patients were recruited at 12 centers across India, 156 patients completed the study and were analyzed. Other demographic characteristics are in Table1.

Table 1: Demographic Characteristics of the patients recruited for the study

\begin{tabular}{|l|c|}
\hline Mean Age of Patients & 9.81 years \\
\hline Males & $82(52 \%)$ \\
\hline Females & $74(48 \%)$ \\
\hline $\begin{array}{l}\text { Patients with Common } \\
\text { Cold/Allergic Rhinitis }\end{array}$ & $134 / 22$ \\
\hline
\end{tabular}

\section{Efficacy Analysis}

TSS was recorded at all the visits (V0, V1 \& V2) and thus the reduction on TSS was calculated. The mean TSS at V0 or the baseline visit was 5.05, which was reduced to 2.58 at V1 or day 3 and further reduced to 0.61 on V2 or day 5 (Figure1). The reduction in TSS corresponds with the improvement in general and physical examination of the patients.

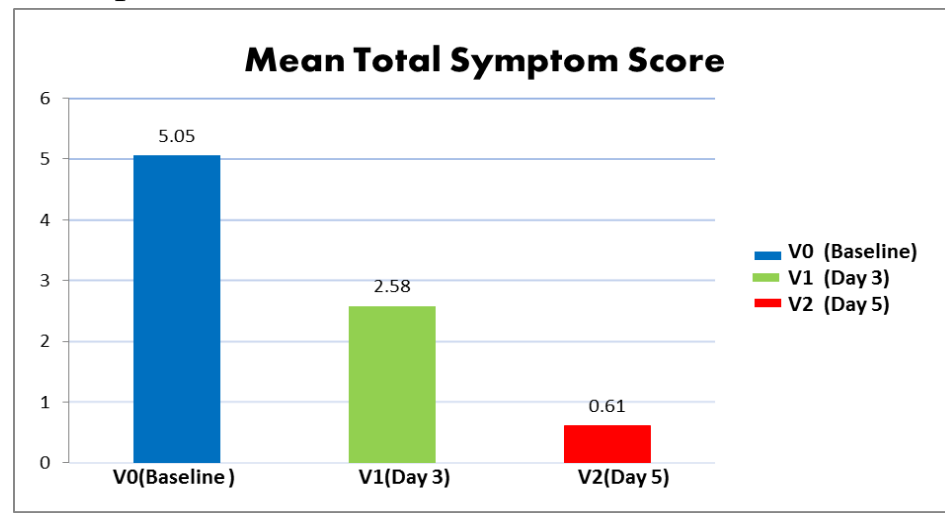

Figure 1: Reduction in TSS at each visit

Extrapolating the data to the Likert-type symptom scale, at V0 or baseline the mean TSS corresponds to Moderate symptoms which was reduced to Mild in V2 or Day 5.

Out of 156 patients, 90 patients had a TSS of 0 i.e.no symptoms on Likert-type symptom scale and another 44 had the TSS of 1(Figure 2) at the end of 5 days.

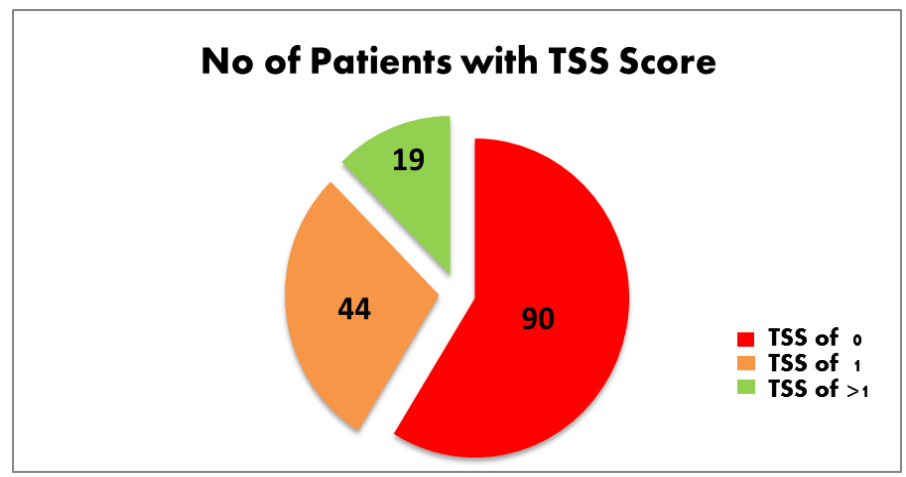

Figure 2: No. of Patients with TSS Score of 0,1 and $>1$ at V2 on day 5

\section{Safety Analysis}

The overall incidence of reported study drug related adverse effects was 20 seen in 21 patients. The list of adverse events with the number of episodes is mentioned in Table2. 
Table 2 : Adverse event episodes occurred in the study

\begin{tabular}{|l|c|}
\hline Adverse Events & No. of Episodes \\
\hline Nausea/Vomiting & $8(40 \%)$ \\
\hline Sedation and Drowsiness & $5(25 \%)$ \\
\hline Hyperacidity & $4(20 \%)$ \\
\hline Palpitation & $2(10 \%)$ \\
\hline Dizziness & $1(5 \%)$ \\
\hline Total & $20 / 156(12.82 \%)$ \\
\hline
\end{tabular}

Majority of adverse effects were study drug related with Nausea/Vomitingdominating, one non-study drug related adverse event was also documented which was shoe bite.

\section{Discussion}

Common cold has a greater impact on productivity, absenteeism, and daily life and since it is treated symptomatically, the treatment is fixated towards symptom control. In author's knowledge this was the first clinical trial conducted to study the efficacy and safety of a fixed-dose combination of Paracetamol, Phenylephrine and Levocetirizine in reducing the symptoms of common cold and allergic rhinitis in Indian children.

Strong arm of this clinical study is that Total Symptom Score (TSS) is used as a criterion for efficacy reckoning and that this data of TSS is extrapolated to Likert-type symptom scale which is the internationally acknowledged scale for common cold symptom assessment. What makes TSS more impressionable is that it has 11 grades for symptom assessment compared to Likert-type symptom scale which has 4 grades, thus increasing the sensitivity of the study.

There was a reduction in Total Symptom Score (TSS) in all the patients in the phase IV post market surveillance study. The TSS reduced from 5.05 to 2.58 in first 3 days which is a reduction of $51.08 \%$ and from 2.58 to 0.61 in the next 2 days which is reduction of $23.64 \%$. The overall reduction in TSS in the 5 days was $87.92 \%$. One point reduction in Likert-type symptom scale from Moderate to Mild took just 3 days with the study drug.In all the patients there was a reduction in the TSS.
Majority of patients had no (TSS score of 0) to very less (TSS score of 1) at the end of 5 study days. Nearly all the patient had more than $50 \%$ reductions in symptoms at every visit.

A total of 20 adverse events were related to study drug. Nausea/Vomiting was the most documented adverse event affecting $5.12 \%$ of study population.

A Cochrane review analysed 32 studies or metaanalysis of 8930 patients for the treatment of Common Cold, construing that antihistamineanalgesic decongestant combinations have some general advantage in adults and older children in treatment of Common Cold ${ }^{10}$. Paracetamol, Phenylephrine and Levocetirizine are specified in the list provided for antihistamine-analgesicdecongestant.

Eccles et al. ${ }^{11}$, theorize the rationale for bringing together multiple drugs in treatment of common cold to provide relief from multiple symptoms. The treatment of common cold by bringing together various drugs to provide relief from multiple symptoms. Furthermore, it is suggested that there is a lack of proof that multi-symptom relief medicines are innately less safe than single active ingredient medicines. Multi-symptom relief combination products containing several active ingredients that provide an effective, safe, economic and convenient option of treating the multiple symptoms of common cold.

Kiran $\mathrm{M}$ et al. ${ }^{12}$ conducted a phase IV study evaluating the efficacy and safety of a combination of Paracetamol, Phenylephrine and Levocetirizine in Treatment of common cold and allergic rhinitis on 201 adult patients.Efficacy assessment was made by curtailment in TSS score which was from 6.82(baseline) to 3.63(day 3) and 1.14 (day 5).The study showed $>50 \%$ reduction in symptom score at all visit and patients in majority had complete relief from the symptom. Thus, the combination of Paracetamol, Phenylepherine and Levocetirizine is safe and effective in the treatment of common cold and allergic rhinitis in adults was concluded. 
Piconet al. ${ }^{6}$, conducted a Phase III clinical study in Brazilian population with a consolidation of Paracetamol, Phenylephrine and Chlorpheniramine maleate (in a different strength capsule) in treatment of Common Cold in adults. Efficacy and safety of the combination were evaluated in 146 patients and were compared with placebo. The curtailment of symptom score in the combination (test) arm was from baseline score of 14.09 to 3.54 at the end of 10 days study period. At the end of 10 days the reduction in placebo arm was from a baseline score of 14.23 to 4.64 . The distribution, type and number of adverse events were analogous in both the groups. The study ceased that the combination of Paracetamol, Phenylephrine and Chlorpheniramine maleate is better than placebo in the treatment of common cold and flu-like syndrome in adults.

Kiran $\mathrm{M}$ et al. ${ }^{13}$ conducted a phase IV clinical study of a combination of Paracetamol, Phenylepherine and Chlorpheniramine maleate essentially in the treatment of common cold. Safety and efficacy of the combination were gauged in 187 patients. Efficacy assessment was made by reduction in Total Symptom Score and four point Likert-type scales with reduction in TSS from 6.58(baseline) to 3.76(day 3) and 1.78 (day 5) showing 50\% reduction in symptom score at all visit. The study concluded that the combination of Paracetamol, Phenylepherine and Chlorpheniramine maleate is safe and effective in the treatment of Common Cold.

The limitation of our study was that common cold being a self-limiting disease, may resolve spontaneously. The cause for reduction in symptoms may not be solely attributed to the study drug. We have tried to minimize this limitation by keeping the study duration for a period of 5 days as opposed to the earlier studies which was for 10 days. Several papers suggest common cold resolves in about 7 to 9 days; ergo the benefit offered on day 5 would be majorly due to the study drug.

\section{Conclusion}

A combination of Paracetamol 250mg, Levocetirizine $1.25 \mathrm{mg}$ and Phenylepherine $5 \mathrm{mg}$ provides optimum symptomatic relief and is safe for the treatment of common cold and allergic rhinitis in children.

\section{Acknowledgements and Disclosures}

Dr. Mayuresh Kiran, Study Director for this study and other authorsare employees of Centaur Pharmaceuticals Pvt. Ltd. This study was conducted as a part of Pharmacovigilance activity for Sinarest LP Syrup manufactured and marketed by Centaur Pharmaceuticals Pvt. Ltd. in accordance with Pharmacovigilance Program of India (PvPI).

\section{References}

1. Worrall G, Del Mar C. There's a Lot of It About: Acute Respiratory Infection in Primary Care. Radcliffe Publishing; 2006.

2. Dicpinigaitis PV, Eccles R, Blaiss MS, Wingertzahn MA. Impact of cough and common cold on productivity, absenteeism, and daily life in the United States: ACHOO Survey. Current medical research and opinion. 2015 Aug 3;31(8):1519-25.

3. Eccles R. Understanding the symptoms of the common cold and influenza. The Lancet infectious diseases. 2005 Nov 30;5(11):718-25.

4. Varshney J, Varshney H. Allergic rhinitis: An overview. Indian Journal of Otolaryngology and Head \& Neck Surgery. 2015 Jun 1;67(2):143-9.

5. Allergic rhinitis and co-morbid asthma: perspective from India -- ARIA AsiaPacific Workshop report.

6. Picon PD, Costa MB, da VeigaPicon R, Fendt LC, Suksteris ML, Saccilotto IC, Dornelles AD, Schmidt LF. Symptomatic treatment of the common cold with a fixed-dose combination of paracetamol, chlorphenamine and phenylephrine: a randomized, placebo-controlled trial. BMC 
infectious diseases. 2013 Nov 22;13(1): 556.

7. Jóźwiak- Bebenista M, Nowak JZ. Paracetamol: mechanism of action, applications and safety concern. Actapoloniaepharmaceutica. 2013 Dec; 71(1):11-23.

8. Walsh, Garry M. "The anti-inflammatory effects of levocetirizine-are they clinically relevant or just an interesting additional effect?." Allergy, Asthma \& Clinical Immunology 5.1 (2009): 14.

9. Meltzer, Eli O., et al. "Treatment of congestion in upper respiratory diseases. "International journal of general medicine 3 (2010): 69.

10. De Sutter, A. I., et al. "Oral antihistaminedecongestant-analgesic combinations for the common cold." Cochrane Database of Systematic Reviews 2.2 (2004).

11. Eccles, Ron, Ingo Fietze, and Uwe-Bernd Rose. "Rationale for treatment of common cold and flu with multi-ingredient combination products for multi-symptom relief in adults." Open Journal of Respiratory Diseases 4.03 (2014): 73.

12. Kiran M, Pawaskar L, Yadav P.'Efficacy and Safety of a combination of Paracetamol, Levocetirizine and Phenylepherine in the symptomatic treatment of Common Cold and Allergic Rhinitis: Phase IV Clinical Study. "International Journal of Current Research in Medical Sciences3.01 (2017):53-59.

13. Kiran M, Pawaskar L, Yadav P.Efficacy and Safety of a combination of Paracetamol, Chlorpheniramine maleate and Phenylepherine in the symptomatic treatment of Common Cold: Phase IV Clinical study. The Indian Practitioner 70.02(2017):10-15. 